\title{
MODIFICATION AND THE COHOMOLOGY GROUPS OF COMPACT SOLVMANIFOLDS
}

\author{
DANIEL GUAN
}

(Communicated by Keith Burns)

\begin{abstract}
In this note we give a modification theorem for a compact homogeneous solvmanifold such that a certain Mostow type condition will be satisfied. An application of this result is a simpler way to calculate the cohomology groups of compact quotients of real solvable Lie group over a cocompact discrete subgroup. Furthermore, we apply the second result to obtain a splitting theorem for compact complex homogeneous manifolds with symplectic structures. In particular, we are able to classify compact complex homogeneous spaces with pseudo-Kählerian structures.
\end{abstract}

\section{INTRODUCTION}

A compact real homogeneous manifold $M=G / H$ is called a solvmanifold if $G$ is solvable and $H$ is discrete. In this paper we always assume that $G$ is connected and simply connected. If $\operatorname{Ad}(G)$ has the same real algebraic closure as that of $\operatorname{Ad}(H)$, we say that $M$ has the Mostow condition with respect to $G$ and $H$. The Mostow condition can also be defined for any given compact homogeneous space. In the general case, the compact factor in the semisimple part has to be the identity. To make things simpler, we only consider the case when $G$ is a real solvable Lie group and $H$ is a cocompact discrete subgroup, i.e., $M$ is a solvmanifold. When $M$ is a solvmanifold and satisfies the Mostow condition, the cohomology of $M$ can be calculated by the cohomology of the Lie algebra (see [Rg, Corollary 7.29]). But in general, it is very difficult to calculate the cohomology of a general compact solvmanifold. In this paper we prove the following results.

Main Theorem 1. If $M=G / H$ is a compact real homogeneous solvmanifold, then there is a finite covering space $M^{\prime}=G / H^{\prime}$; i.e., $H / H^{\prime}$ is a finite group such that there is another simply connected solvable real Lie group $G^{\prime}$ which contains $H^{\prime}$ and is diffeomorphic to $G$ such that

(1) $M^{\prime}=G^{\prime} / H^{\prime}$;

(2) $M^{\prime}$ satisfies the Mostow condition with respect to $G^{\prime}$ and $H^{\prime}$.

In particular, the following theorem holds.

Received by the editors August 10, 2006 and, in revised form, October 11, 2007.

2000 Mathematics Subject Classification. Primary 53C15, 57S25, 53C30, 22E99, $15 \mathrm{~A} 75$.

Key words and phrases. Solvmanifolds, cohomology, invariant structure, homogeneous space, product, fiber bundles, symplectic manifolds, splittings, prealgebraic group, decompositions, modification, Lie group, compact manifolds, uniform discrete subgroups, locally flat parallelizable manifolds. 
Main Theorem 2. If $G$ is solvable, $H$ is discrete, and $M=G / H$ is compact, then we have $H^{*}(M)=H^{*}\left(\mathcal{G}^{\prime}\right)$, where $\mathcal{G}^{\prime}$ is the Lie algebra of the Lie group $G^{\prime}$ in the Main Theorem 1.

A smooth $2 n$-dimensional manifold $M$ equipped with a smooth transitive action of a Lie group is called a homogeneous space. If in addition $M$ is a symplectic manifold, we refer to it as a homogeneous space with a symplectic structure and, if the structure is invariant, a homogeneous space with an invariant symplectic structure.

Recently there has been much progress in the area of symplectic manifolds and group actions. I was interested in the classical problem of classifying compact homogeneous spaces with symplectic structure. The main difficulty in this problem is that we do not know anything about the transitive group and the isotropy group (cf. [DG, Hk, Gu3]). In the Kähler case we know that the isometric group is compact. In an earlier paper [Gu3] the following theorem is proved.

Proposition 1. Every finite-dimensional Lie subgroup of the automorphism group of a compact symplectic manifold is locally a product of a compact semisimple group and a 2-step solvable group $R$. Moreover, the adjoint representation of $R$ on $R^{\prime}$ is a subgroup of a compact torus.

I am also interested in the structure of a compact homogeneous manifold with a symplectic structure (which might not be invariant under the group action).

The following conjecture was posed in [Gu3].

Conjecture. If $G / H$ is a compact homogeneous space with a symplectic structure, then $G / H$ is diffeomorphic to a product of a rational homogeneous space and another homogeneous space $N$, where, up to a finite quotient, $N$ is a compact quotient of a compact locally flat parallelizable manifold with a symplectic structure.

Our Main Theorem 1 will be a major step toward a proof of this conjecture. We call a manifold $N$ locally flat parallelizable if $N=G / H$ for a simply connected Lie group $G$ which is diffeomorphic to $\mathbf{R}^{k}$ for some integer $k$ and $H$ is a uniform (i.e., cocompact) discrete subgroup.

In this paper, we will prove this conjecture in the complex case. Namely, we will prove the following theorem.

Main Theorem 3. If $M=G / H$ is a compact complex homogeneous space with a symplectic structure and $G$ is complex (we can always assume this), then $M$ is a product of a rational projective homogeneous space and a complex solvmanifold $N=N_{G}\left(H^{0}\right) / H$. Moreover, if we let $G_{1}=N_{G}\left(H^{0}\right) / H^{0}$ and $\Gamma=H / H^{0}$, then there is a subgroup $\Gamma^{\prime}$ of $\Gamma$ such that $\Gamma / \Gamma^{\prime}$ is finite and $N$, up to a finite quotient, is a solvmanifold $G_{2} / \Gamma^{\prime}$ with a right invariant symplectic structure of a real Lie group $G_{2}$ which contains the nilradical of $G_{1}$ and is possibly different from $G_{1}$. In particular, if $M$ has a pseudo-Kählerian structure, so does $N$, and if $N$ has a right invariant pseudo-Kählerian structure, $N$ must be a complex torus. Also, if $M$ has a holomorphic symplectic structure, then $M=N$.

We note that for the splitting theorem from [Gu1, Gu2] to hold, we only need to have a real symplectic structure which is invariant under the maximal compact subgroup and this is provided by the existence of $G^{\prime}$ in our Main Theorems 1 and 2 . Also, the complex structure is right invariant under the action of $G_{2}$; see Corollary 1 in the next section. 
The case where $M=N$ has a right invariant pseudo-Kählerian metric was proven in [Gu1, Gu2] (see [Gu2, proof of Lemma 1] or much earlier work [DG, Section 3.3]). The theorem in $[D G]$ follows also from our Main Theorem 3. Applying the method of [DG] to $G_{2}$, one can easily prove that if $N$ is pseudo-Kählerian, then the nilradical $N_{G_{1}}$ of $G_{1}$ has at most two steps. An application of the Leray spectral sequence to the fiber bundle $G_{2} / \Gamma^{\prime} \rightarrow G_{2} / N_{G_{2}} \Gamma^{\prime}$ shows that the derivator $\left[N_{G_{2}}, N_{G_{2}}\right]=0$; see Corollary 2 in the next section.

Moreover, the Lie algebra of $G_{2}$ is a direct orthogonal sum of two vector spaces $A$ and $N_{G_{2}}$ (regarded as its Lie algebra) with respect to a pseudo-Kählerian structure $\omega$ which is right invariant on the universal covering. In addition, both $\omega_{1}=\left.\omega\right|_{A}$ and $\omega_{2}=\left.\omega\right|_{N_{G_{2}}}$ are closed, and are nonzero cohomology classes. $A$ is an Abelian Lie algebra and acts on $N_{G_{2}}$ semisimply with real eigenvalues. The structure $\omega_{2}$ has zero index. This is done with a new algebra called complex-parallelizable-rightinvariant-pseudo-Kählerian algebra, which is similar to what we used in [DG, Gu3] (see the proof of Corollary 1 in the next section). We should deal with the pseudoKählerian case in another paper [Gu4]. One might also notice that when $\omega$ is Kähler, then $\omega_{2}=0$ and we have the Borel-Remmert Theorem.

When the pseudo-Kählerian structure is Kähler, the original average process in [Gu1, Gu2] works and gives an averaged Kähler structure. Then the splitting theorem holds there and an application of our argument there produces another proof of the Borel-Remmert Theorem.

The main purpose of [Gu1] was finding non-Kählerian holomorphic symplectic manifolds. We would like to note that Theorem $B$ from [Gu1, Gu2] is still true even though the proof was not correct. ${ }^{1}$ From our Main Theorem 3 it follows that for the complex parallelizable manifold $M=N$ the holomorphic tangent bundle is trivial and therefore $H^{2,0}(M)=\wedge^{2} \mathcal{G}^{*}$, which all come from the right invariant forms. Therefore, the averaging process goes through trivially.

\section{THE PROOF}

2.1. We will need some results from the splitting theory of the Lie group (see [Gb1]). Let $G=S R$ be a Levi decomposition of a semisimple Lie group. In this section, we assume that $G$ is simply connected. We call $G$ a splittable Lie group if $R=T U$ where $T \cap U=\{e\}, T$ acts semisimply and $U$ acts unipotently on the Lie algebra $\mathcal{G}$. We call a Lie group embedding $\alpha: G \rightarrow M(G)$ from $G$ to a splittable simply connected Lie group $M(G)=T \cdot S \cdot U$ a Mal'cev splitting or M-splitting if $\alpha(G)$ is a normal subgroup of $M(G), M(G)$ is a semidirect product of $T$ and $\alpha(G)$, and $\alpha(G) \cdot U=M(G)$.

Proposition 2. For any simply connected Lie group $G$ there is a unique Mal'cev splitting.

The Mal'cev splitting can be constructed as following:

\footnotetext{
${ }^{1} \mathrm{I}$ tried to prove a stronger version of our Main Theorem 3 with an averaging method in [Gu1, Gu2] about 10 years ago. I did not realize that there was a mistake in [Gu2] until I read [Ym] in August 2006; the author did not tell me. This is unfortunately an odd situation. However, this eventually turned out positively. Thanks to Gorbatsevich's construction I wrote down our Main Theorem 1 in a few days and was able to go around Iwamoto's result, which I had earlier misused, and prove a weaker version, i.e., our Main Theorem 3. The examples in [Ym] gave strong and beautiful evidence for our Main Theorem 3. Finally, I received a letter from Yamada with several of his offprints on April 24, 2007.
} 
Let $G=S \cdot R$ be the Levi decomposition of a connected simply connected Lie group $G$. Consider the adjoint representation $\operatorname{Ad}_{G}: G \rightarrow G L(\mathcal{G})$; put $G^{*}=$ $\operatorname{Ad}_{G}(G)$, and let $\langle G\rangle$ be the algebraic closure of $G^{*}$ in $G L(\mathcal{G})$. Since $\langle G\rangle$ is algebraic, it has a Chevalley decomposition

$$
\langle G\rangle=T^{*} S^{*} U^{*},
$$

where $U^{*}$ is the unipotent radical, $S^{*}$ is semisimple, and $T^{*}$ is Abelian and consists of semisimple (i.e., completely reducible) elements. Put $W^{*}=S^{*} U^{*}$; then $\langle G\rangle=T^{*} W^{*}$, with $T^{*} \cap W^{*}$ finite. Let $t^{*}: T^{*} W^{*} \rightarrow T^{*} / T^{*} \cap W^{*}$ be the natural epimorphism, with kernel $W^{*}$. Writing $\hat{T}=T^{*} / T^{*} \cap W^{*}$, we clearly have $t^{*}(\operatorname{Ad} G) \subset(\hat{T})^{0}$, since $G$ is connected. If for the connected Abelian Lie group $\left(T^{*}\right)^{0}$ we consider the universal covering $\pi_{T}: \tilde{T} \rightarrow\left(T^{*}\right)^{0}$; it is obvious that $t^{*} \cdot \pi_{T}: \tilde{T} \rightarrow(\hat{T})^{0}$ is the universal covering for $(\hat{T})^{0}$. Since $G$ is connected and simply connected, there exists a unique homomorphism $\tilde{t}: G \rightarrow \tilde{T}$ such that $t^{*} \cdot \pi_{T} \cdot \tilde{t}=t^{*} \cdot \operatorname{Ad}_{G}$. Put $T=\tilde{t}(G), T_{G}^{*}=\pi_{T} \cdot \tilde{t}(G)$; then $T$ is a connected simply connected Abelian Lie group covering of $T^{*}$, while $T_{G}^{*} \subset\langle G\rangle$. We see that $T_{G}^{*}$ can be regarded as a subgroup of Aut $G$. The imbedding $T_{G}^{*} \rightarrow$ Aut $G$ and the homomorphism $\pi_{T}$ induce a homomorphism $\phi: T \rightarrow \operatorname{Aut} G$, with $\operatorname{ker} \phi=\operatorname{ker} \pi_{T} \cap T$ discrete. Then we can get the Mal'cev splitting

$$
M(G)=T \times_{\phi} G
$$

and

$$
M(G)=T S U
$$

for a unipotent group $U$ such that

$$
\operatorname{dim} U=\operatorname{dim} R, \quad \operatorname{dim} U / N_{R}=\operatorname{dim} T,
$$

where $N_{R}$ is the nilpotent radical of $G$.

Now, we let

$$
W_{G}=S U, \quad W_{G, l}=S / l(S) \cdot U,
$$

where $l(S)$ is the minimal discrete subgroup of the center of $S$ such that $S_{l}=S / l(S)$ is linear. Then, both Aut $W_{G, l}$ and the semidirect product Aut $W_{G, l} \propto W_{G, l}$ are prealgebraic groups. As $W_{G}$ is a normal subgroup containing the commutator of $M(G)$, we can regard $T_{G}^{*}$ as a subgroup of Aut $W_{G, l}$. Let $a\left(T_{G}^{*}\right)$ be the prealgebraic hull of $T_{G}^{*}$ in Aut $W_{G, l}$, and

$$
\mathcal{A}_{l}(G)=a\left(T_{G}^{*}\right) \propto W_{G, l} .
$$

We see that $\mathcal{A}_{l}(G)$ is prealgebraic. Let $M_{l}(G)=T_{G}^{*} S_{l} U$ as a quotient of $M(G)$. Then we have the following.

Proposition 3. The group $\mathcal{A}_{l}(G)$ is prealgebraic, and there exists an imbedding $\beta: M_{l}(G) \rightarrow \mathcal{A}_{l}(G)$ such that the following properties hold:

(1) $\mathcal{A}_{l}(G)$ is splittable, and if $\mathcal{A}_{l}(G)=T^{\prime} S^{\prime} U^{\prime}$, where $U^{\prime}$ is unipotent, $S^{\prime}$ is semisimple and $T^{\prime}$ is a prealgebraic torus, then $\beta\left(M_{l}(G)\right) \supset S^{\prime} U^{\prime}$ and $S^{\prime}=$ $S_{l}$, where $S$ is the semisimple part of $G$ and $U^{\prime}=U$.

(2) The prealgebraic closure of each of the subgroups $\beta\left(G_{l}\right)$ and $\beta\left(M_{l}(G)\right)$ in $\mathcal{A}_{l}(G)$ is $\mathcal{A}_{l}(G)$ itself. 
Consider a very simple example. Let $G=G_{1} \times G_{2}, G_{1}=T N$ with $T, N, G_{2}$ Abelian, and $T$ acts on $N$ almost faithfully as a compact torus with no eigenvectors. Then $\langle G\rangle=\operatorname{Ad}_{G}(T) N, W^{*}=N$,

$$
\begin{gathered}
t^{*}: \operatorname{Ad}_{G}(T) N \rightarrow \operatorname{Ad}_{G}(T)=\hat{T}, \\
\pi_{T}: T \rightarrow \operatorname{Ad}_{G}(T), \\
\tilde{t}: T N \times G_{2} \rightarrow T, \\
T_{G}^{*}=\operatorname{Ad}_{G}(T), \quad \phi: T \rightarrow \operatorname{Ad}_{G}(T), \\
M(G)=T \times_{\phi} G=T U, \quad U=\left\{\left.\left(t, t^{-1}, n, g\right)\right|_{t \in T} n \in N \quad g \in G_{2}\right\}, \\
W_{G}=W_{G, l}=U, \quad \mathcal{A}_{l}(G)=M_{l}(G)=\operatorname{Ad}_{G}(T) U .
\end{gathered}
$$

2.2. Here we prove the modification of our Main Theorem 1 for a compact homogeneous solvmanifolds. The modification method was first used in [Gb2]. Similar construction can be found in the study of homogeneous Kähler manifolds, e.g., [Dm], [DN].

In this subsection we only deal with the case when $G$ is solvable, i.e., $S$ is the identity and $H$ is discrete, in particular $H^{0}$ is also the identity.

Let $M=G / H$ be a compact homogeneous solvmanifold of a simply connected solvable real Lie group $G$. We go through the proof with similar notation as in [Gu3, 3.1.7], which might help us understand both constructions here and therein. In our case, we let $G_{*}=G_{l}=G / l(S)=G$ be the image of $G$ (and $H_{*}=H / H \cap l(S)=H$ be the image of $H)$ in $\mathcal{A}_{l}(G)=\mathcal{A}(G)$. We also let $P_{*}$ be the algebraic closure of $\beta(H)$ in $\mathcal{A}(G)$. Then the fact that nilradical $N_{R} \subset P_{*}$ follows from a theorem proved by Mostow [Rg, Theorem 3.3]; see also [Rg, Corollary 8.27, 8.28]. In [Gu3, Theorem 3.1.7] this condition was true because the nilradical is in the normalizer of $H^{0}$, which of course is trivial in our case here since $H^{0}$ is the identity. We see that our modification is quite different from the one mentioned in [Gu3]). Since the subgroup $P_{*}$ is an algebraic group, the group $\pi_{0}\left(P_{*}\right)$ is finite. Passing from $H$ to the subgroup $H_{1}=H \cap \pi^{-1}\left(P_{*}^{0} \cap H_{*}\right)$ of finite index, where $\pi: M(G) \rightarrow M_{l}(G)=M(G)$ is the natural epimorphism, we might assume that $H_{*} \subset P_{*}^{0}$ by considering a finite covering $M^{\prime}$ of $M$. This inclusion will be assumed to hold in what follows.

We consider the natural epimorphism $\gamma: \mathcal{A}_{l}(G) \rightarrow \mathcal{A}_{l}(G) / W_{l}$. We have

$$
\mathcal{A}_{l}(G) / W_{l}=T_{*} \times \pi\left(W_{G}\right) / W_{l}
$$

with $W_{G}=S U=U, W_{l}=S_{l} N_{R}=N_{R}$ (our $W_{l}$ is the same as in [Gb2] but different from the one in [Gb1]; in [Gb1] $\left.W_{l}=S / l(S) \cdot U=U\right)$ and $T_{*}$ is a prealgebraic torus; $\pi\left(W_{G}\right) / W_{l}=U / N_{R}$. So $\operatorname{Im} \gamma=T_{*} \times U / N_{R}$, and we denote it by $A ; A$ is connected and Abelian. There is a natural embedding of the group $G_{*} / W_{l}=R / N_{R}$ in $M_{l}(G) / W_{l}$, which is contained in $A$.

We denote the image of $R / N_{R}$ by $B$. We have that $U / N_{R}$ is the projection of $B$ to the second factor $U / N_{R}$. By our construction we have $\operatorname{dim} U / N_{R}=\operatorname{dim} R / N_{R}$. The composition of $\gamma$ and the projection restricted on $R / N_{R}$ is a linear map from one Euclidean space onto another of the same dimension, and therefore is an isomorphism. That implies $B \cap T_{*}=\{e\}$. We see that the projection $\mu: T_{*} \times U / N_{R} \rightarrow U / N_{R}$ to the second factor is an isomorphism on $B$, i.e., $B$ is closed in $A$. Now we consider the subgroup $H_{*} / H_{*} \cap W_{l}$ of $A$ and its closure $\overline{H_{*} / H_{*} \cap W_{l}}$ (in the Euclidean topology), which we denote by $A_{1}$. Since $H_{*} / H_{*} \cap W_{l} \subset B$, we have $A_{1} \subset B$. Since 
the group $B$ is simply connected and Abelian, $A_{1}$ is a closed subgroup of it, and $A_{1}$ is torsion free and isomorphic to $\mathbf{R}^{p} \times \mathbf{Z}^{q}$ for some $p, q \geq 0$.

Finally we consider the subgroup $\gamma\left(P_{*}\right) \subset A$. The subgroup $\operatorname{Ker} \gamma=W_{l}=N_{R}$ is closed in the Zariski topology on $\mathcal{A}_{l}(G)$, and so is the Lie group $P_{*}$; therefore $\gamma\left(P_{*}\right)$ is a closed subgroup of $A$. But $H_{*} \subset P_{*}$, so $H_{*} / H_{*} \cap W_{l} \subset \gamma\left(P_{*}\right)$ and hence $A_{1} \subset \gamma\left(P_{*}\right)$, i.e., $A_{1} \subset \gamma\left(P_{*}^{0}\right)$ by our convention. The group $\gamma\left(P_{*}^{0}\right)$ is connected and Abelian and hence $\gamma\left(P_{*}^{0}\right)=K \times V$, where $K$ is a maximal compact subgroup of $\gamma\left(P_{*}^{0}\right)$ (which is a torus), and $V$ is simply connected. Since $A_{1}$ is closed in $A$ and torsion free, $A_{1} \cap K=\{e\}$. Hence the projection $K \times V \rightarrow V$ onto the second direct factor is a monomorphism on $A_{1}$. Now it follows from this that there exists a closed simply connected subgroup $C \subset \gamma\left(P_{*}^{0}\right)$ such that $A_{1} \subset C$ and $A_{1}$ is uniform in $C$ (we note that $C$ is not always in $B$ ). We set $\Phi_{l}=\gamma^{-1}(C)$. Then $\Phi_{l} \subset P_{*}^{0}$ and therefore has the same algebraic closure as $H_{l}$. Then $\Phi_{l}$ is a closed connected subgroup of $\mathcal{A}_{l}(G)$. Corresponding to it is a closed connected subgroup $\Phi$ of $\mathcal{A}(G)$.

With this construction at hand, we have the following theorem.

Theorem. Let $M=G / H$ be a compact homogeneous solvmanifold of a simply connected Lie group $G$. Then there exists a subgroup $H^{\prime}$ of finite index in $H$ and a subgroup $\Phi$ of $\mathcal{A}(G)$, such that:

(a) $\Phi$ is a connected, simply connected, closed subgroup of $\mathcal{A}(G)$, containing $H^{\prime}$ and $N_{R}$;

(b) $W_{\Phi}=W_{G}=U_{\Phi}=U_{G}$ (although $M(\Phi)$ and $M(G)$ are not generally isomorphic);

(c) for the decomposition $\mathcal{A}(G)=T W_{G}=T U$ with $T$ an Abelian subgroup of $\mathcal{A}(G)$ we have $\Phi \subset T G, G \subset T \Phi$, where $\Phi \cap T=G \cap T=\{e\} ;$

$(d)$ there exists a diffeomorphism $\eta: \Phi \rightarrow G$ which is the identity on the subgroup $H^{\prime}$ and induces a diffeomorphism $\Phi / H^{\prime} \rightarrow G / H^{\prime}$. Actually, it induces a diffeomorphism of the torus bundles $G / H^{\prime} \rightarrow G / H^{\prime} N_{R}$ and $\Phi / H^{\prime} \rightarrow \Phi / H^{\prime} N_{R}$

(e) $\operatorname{Ad} \Phi$ has the same algebraic closure as that of $\mathrm{Ad} H^{\prime}$.

We obtain our Main Theorem 2 by applying the Mostow Theorem (see [Rg, Corollary 7.29]). One might notice that our proof here is almost word-for-word identical to the corresponding parts 3.1.6 and 3.1.7 in [Gu3], but the purposes are quite different. For the proof of Main Theorem 3, one can easily apply Main Theorem 2 to $N$ and follow the proof in our previous work [Gu2].

Also, we point out that in the real case one could use $\langle G\rangle$ instead of $\mathcal{A}(G)$, but no argument for a mathematical proof using $\langle G\rangle$ is available at the present time. The proof of the next result also shows that using $\mathcal{A}(G)$ is better.

Moreover, since $G_{1}$ is a complex Lie group, at the point $e$ we have

$$
\operatorname{Ad}(g) \cdot J=J \cdot \operatorname{Ad}(g)
$$

for any $g \in G_{1}$. Therefore, we also have

$$
\alpha \cdot J=J \cdot \alpha
$$

for any $\alpha \in\left\langle G_{1}\right\rangle$. Now we let

$$
j(x+t)=J x\left(\bmod \operatorname{Lie}\left(\left\langle G_{1}\right\rangle\right)\right),
$$


where $\operatorname{Lie}\left(\left\langle G_{1}\right\rangle\right)$ is the Lie algebra of $\left\langle G_{1}\right\rangle$. One could easily check that

$$
\begin{aligned}
& {[j(x+t), j(y+s)]=-[x, y]} \\
& \quad=-[x, y]+j t(j y)+t(y)-s(x)-j s(j x)+[t, s]\left(\bmod \operatorname{Lie}\left(\left\langle G_{1}\right\rangle\right)\right) \\
& \quad=j[j(x+t), y+s]+j[x+t, j(y+s)]+[x+t, y+s]
\end{aligned}
$$

for any $x, y \in \mathcal{G}_{1}, s, t \in \operatorname{Lie}\left(\left\langle G_{1}\right\rangle\right)$. This means that the complex structure on $G_{1}$ is always invariant under the action of $M\left(G_{1}\right)=T \times{ }_{\phi} G$ and hence its prealgebraic closure $\mathcal{A}\left(G_{1}\right)$; see $[\mathrm{Dm}, \mathrm{DN}, \mathrm{DG}]$ for this formula.

Corollary 1. The complex structure on $N$ of Main Theorem 3 is right invariant under the action of $G_{2}$.

Now any element in $H^{1}\left(N_{G_{1}} / \Gamma \cap N_{G_{1}}, \mathbf{R}\right)$ is $\beta+\bar{\beta}$ with a holomorphic left invariant form $\beta$ which is closed, that is, $d(\omega)=0$. This means that $\beta$ is not from the derivation $\left[N_{G_{1}}, N_{G_{1}}\right]$ but from $N_{G_{1}} /\left[N_{G_{1}}, N_{G_{1}}\right]$ (see [Gu1]). So a right invariant pseudo-Kählerian form on $G_{2} / \Gamma^{\prime}$ actually comes from $M_{1}=G_{2} /\left[N_{G_{2}}, N_{G_{2}}\right] \Gamma^{\prime}$. Therefore, combined with what we have for the part from $H^{2}\left(G_{2} / \Gamma^{\prime}, \mathbf{R}\right)$ in $[\mathrm{DG}]$, we get all the three parts in the Leray spectral sequence come from $M_{1}$ (see a similar argument in [Gu2]). By the nondegeneracy of the pseudo-Kählerian form we see that $N=M_{1}$.

Corollary 2. If $M$ is a pseudo-Kählerian manifold in Main Theorem 3, then $N$ is a complex torus bundle over a complex torus and has, up to a finite covering, a left $G_{2}$ invariant pseudo-Kählerian structure. Moreover, $G_{1}$ and $G_{2}$ have Abelian nilradicals.

These compact complex homogeneous parallelizable manifolds also show that the similar result of the Mostow Theorem for the Dolbeault cohomology does not work in general, since the holomorphic 1-forms (as elements in $H^{1,0}(N)$ ) are generally not right invariant under the modified Lie group which satisfies the Mostow condition.

With some extra work we can prove that all compact complex homogeneous spaces with pseudo-Kählerian structure have the similar form as in Yamada's example [Gu4].

\section{REFERENCES}

[Dm] J. Dorfmeister: Homogeneous Kähler Manifolds Admitting a Transitive Solvable Group of Automorphisms, Ann. Scient. Ec. Norm. Sup., 4 Ser., 18 (1985), 143-180. MR803198 $(87 \mathrm{j}: 32094)$

[DN] J. Dorfmeister \& K. Nakajima: The Fundamental Conjecture for Homogeneous Kähler Manifolds, Acta Math. 161 (1988), 23-70. MR962095 (89i:32066)

[DG] J. Dorfmeister \& Z.-D. Guan: Classification of Compact Homogeneous Pseudo-Kähler Manifolds, Comment. Math. Helv. 67 (1992), 499-513. MR1185806 (93i:32042)

[Gb1] V. V. Gorbatsevich: Splittings of Lie Groups and Their Application to the Study of Homogeneous Spaces, Math. USSR Izv. 15 (1979), 441-467. MR0567035 (82e:22019)

[Gb2] V. V. Gorbatsevich: Plesiocompact Homogeneous Spaces, Siber. Math. J. 30 (1989), 217226. MR997468 (90f:22010)

[Gu1] Z. Guan: Examples of compact holomorphic symplectic manifolds which admit no Kähler structure. In Geometry and Analysis on Complex Manifolds-Festschrift for Professor S. Kobayashi's 60th Birthday, World Scientific 1994, pp. 63-74. MR1463964 (98h:53109)

[Gu2] D. Guan: A Splitting Theorem for Compact Complex Homogeneous Spaces with a Symplectic Structure, Geom. Dedi. 67 (1996), 217-225. MR1413633 (98a:53105)

[Gu3] D. Guan: On Compact Symplectic Manifolds with Lie Group Symmetries, Transactions of AMS 357 (2005), 3359-3373. MR2135752 (2006c:53094) 
[Gu4] D. Guan: Classification of Compact Complex Homogeneous Manifolds with PseudoKählerian Structures, Preprint, 2007.

[Hk] A. Huckleberry: Homogeneous Pseudo-Kählerian Manifolds: A Hamiltonian Viewpoint, Note Mat. 10 (1990), 337-342. MR1221949 (94f:53052)

[Rg] M. S. Raghunathan: Discrete Subgroups of Lie Groups, Springer-Verlag, Berlin, 1972. MR0507234 (58:22394a)

[Ym] T. Yamada: A Pseudo-Kähler Structure on a Nontoral Compact Complex Parallelizable Solvmanifold, Geom. Dedicata 112 (2005), 115-122. MR2163892 (2006e:53061)

Department of Mathematics, University of California at Riverside, Riverside, CA 92507

E-mail address: zguan@math.ucr.edu 